\title{
Flow-Pattern Details in an Aneurysm Model Using High-Speed 1000-Frames-per-Second Angiography
}

\author{
(D).M. Krebs, (D) A. Shankar, (D) S.V. Setlur Nagesh, DJ.M. Davies, (D).V. Snyder, (DE.I. Levy, (D).N. Hopkins, (D) M. Mokin,
}

(DD.R. Bednarek, DA.H. Siddiqui, and (D). Rudin

\begin{abstract}
SUMMARY: Traditional digital subtraction angiography provides rather limited evaluation of contrast flow dynamics when studying and treating intracranial brain aneurysms. A 1000-frames-per-second photon-counting $x$-ray detector was used to image detailed iodinecontrast flow patterns in an internal carotid artery aneurysm of a 3D-printed vascular phantom. High-speed imaging revealed differences in vortex and inflow patterns with and without a Pipeline Embolization Device flow diverter in more detail and clarity than could be seen in standard pulsed angiography. Improved temporal imaging has the potential to impact the outcomes of endovascular interventions by allowing clinicians to better understand and act on flow dynamics in real-time.
\end{abstract}

ABBREVIATION: $\mathrm{fps}=$ frames per second

$\mathrm{H}$ emodynamics are important in determining intervention outcomes. Conventional flat panel $\mathrm{x}$-ray detectors operating at 30-60 frames per second (fps) offer limited visualization of aneurysmal flow patterns due to insufficient temporal resolution. Instead, studies seeking to understand the hemodynamics of intracranial arteries currently use computational fluid dynamics, ${ }^{1}$ with complex algorithms to recreate flow patterns as opposed to directly measuring them. Although computational fluid dynamics is excellent for the display of detail in hemodynamics, it is an approximation of flow conditions and requires a long computational time to run the simulations, which significantly limits real-time applicability. We herein present a new method of using a new high-speed $\mathrm{x}$-ray detector to visu-

Received January 24, 2019; accepted after revision April 25.

From the Canon (formerly Toshiba) Stroke and Vascular Research Center (J.M.K., A.K., S.V.S.N., K.V.S., E.I.L., L.N.H., D.R.B., A.H.S., S.R.) and Departments of Biomedical Engineering (S.R.), Mechanical and Aerospace Engineering (S.R.), and Electrical Engineering (S.R.), University at Buffalo, State University of New York, Buffalo, New York; Department of Neurosurgery (J.M.D., K.V.S., E.I.L., L.N.H., A.H.S., S.R.), Gates Vascular Institute at Kaleida Health, Buffalo, New York; Departments of Neurosurgery (S.V.S.N., J.M.D., K.V.S., E.I.L., L.N.H., D.R.B., A.H.S., S.R.), Bioinformatics (J.M.D.), Radiology (E.I.L., L.N.H., D.R.B., A.H.S., S.R.), and Neurology (K.V.S.), Jacobs School of Medicine and Biomedical Sciences, University at Buffalo, State University of New York, Buffalo, New York; Jacobs Institute (J.M.D., K.V.S., E.I.L., L.N.H., A.H.S.), Buffalo, New York; and Department of Neurosurgery and Brain Repair (M.M.), University of South Florida, Tampa, Florida.

This work was partially supported by a research grant from Canon Medical Systems Corporation.

Please address correspondence to Stephen Rudin, PhD, Canon (formerly Toshiba) Stroke and Vascular Research Center, University at Buffalo, 875 Ellicott St, Buffalo, NY 14203; e-mail: srudin@buffalo.edu

Indicates article with supplemental on-line videos.

http://dx.doi.org/10.3174/ajnr.A6090 alize the details of arterial hemodynamics during an interventional procedure.

Aneurysms are abnormal outpouchings on arteries, and 1 treatment option involves the use of flow-diverting stents, such as the Pipeline Embolization Device (PED; Covidien, Irvine, California). Flow diverters induce a modification of blood flow within and around the inflow zone of an aneurysm that leads to gradual intra-aneurysmal thrombosis and healing. During the intervention, clinicians must make critical judgements regarding placement, repositioning, and stacking of flow diverters that will ultimately impact treatment efficacy 6 months to a year later; thus, a detailed understanding of how flow is altered could critically impact treatment. In this work, we present the use of a 1000-fps single-photon-counting $\mathrm{x}$-ray detector to visualize changes in detailed flow patterns in and around a 3D-printed flow-divertertreated internal carotid artery aneurysm model in high spatial and temporal resolution.

\section{MATERIALS AND METHODS \\ Detector Description}

The Actaeon detector (XCounter, Danderyd, Sweden) is a singlephoton-counting direct cadmium telluride detector with a $100-\mu \mathrm{m}$ pixel pitch and a $256 \times 256$ matrix size, capable of acquisition speeds up to $1000 \mathrm{fps}^{2}$

\section{Aneurysm Model Description}

The process for fabricating 3D-printed models has been previously described. ${ }^{3}$ The model, simulating an internal carotid artery aneurysm, was connected to a pulsatile flow loop with water used 


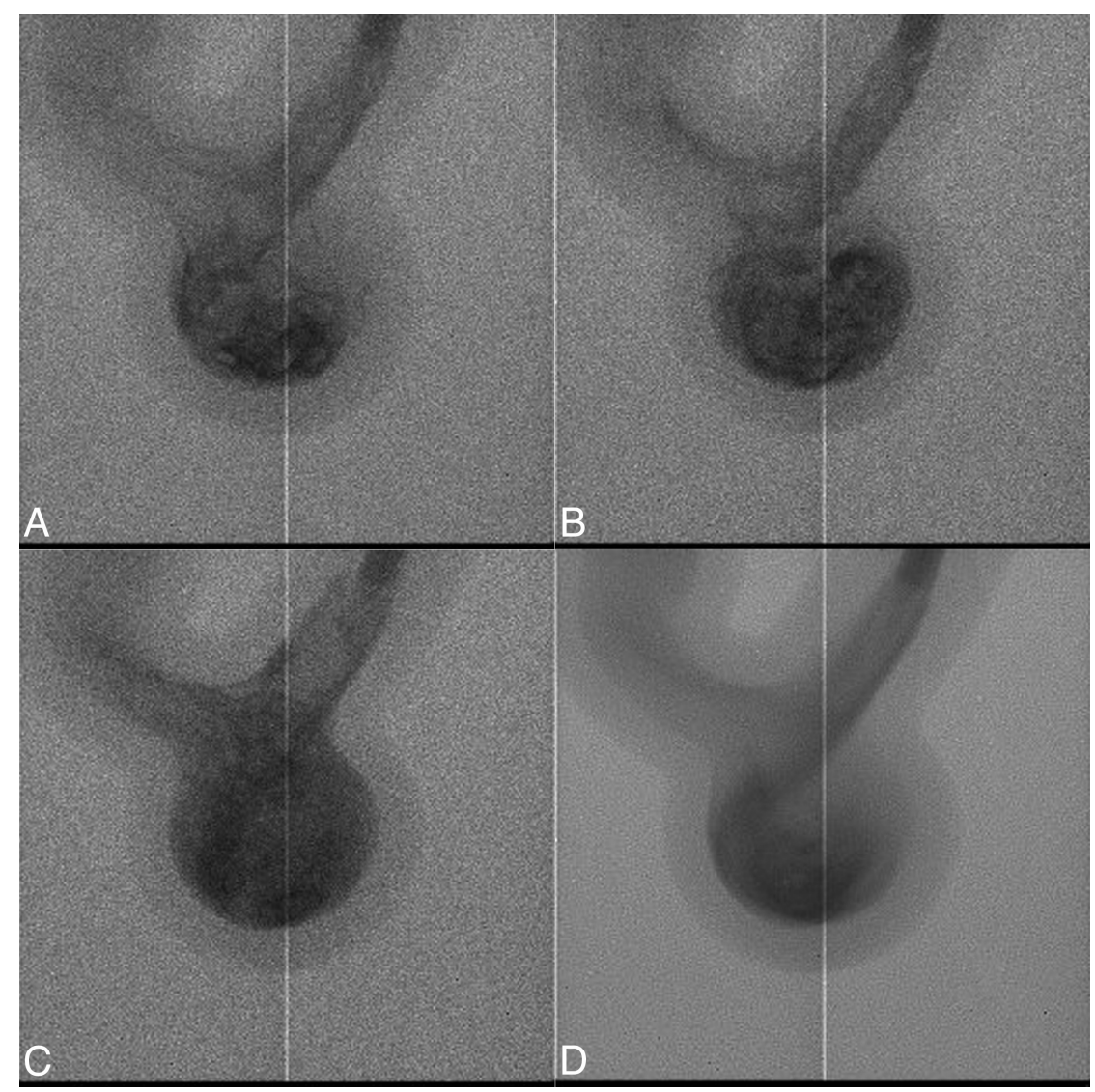

FIG 1. DSA images show flow detail in an internal carotid artery aneurysm model when no PED flow diverter is deployed, acquired at $1000 \mathrm{fps}$ and separated by 25 frames each $(A-C)$, and a single averaged image for one 40-ms $x$-ray pulse $(D)$. The vertical line shows the 1-pixel boundary between detector modules. For the full sequence, see On-line Video 1.

as the circulating fluid. A 21-mm aluminum block was added underneath the model to approximate the $\mathrm{x}$-ray attenuation of a human head (RQA5 attenuator, IEC 61267-1, 62220-1).

\section{Image Acquisition}

The detector was mounted on a Unistrut platform (Unistrut, Harvey, Illinois) and positioned $6 \mathrm{~cm}$ above the phantom, resulting in a magnification factor of 1.08 , and was set to acquire continuously at $1000 \mathrm{fps}$. An Infinix biplane C-arm system (Model INFX-8000V; Canon, Otawara, Japan) was used as the $\mathrm{x}$-ray source. Peak tube voltage was set to $92 \mathrm{kV}$, the tube current was set to $160 \mathrm{~mA}$, and the exposure pulse width was set to $100 \mathrm{~ms}$ with a medium focal spot. A 6F catheter (Model 7512-23; Merit Medical, South Jordan, Utah) was placed proximal to the aneurysm region. With the catheter in place, an AutoSyringe (Model PPD11060507; Medrad, Warrendale, Pennsylvania) was used to inject a $10-\mathrm{mL}$ burst of $350 \mathrm{mg}$ of undiluted iodine per milliliter of contrast agent into the $3 \mathrm{D}$ printed vessel at $10 \mathrm{~mL} / \mathrm{s}$. The delay between the injection and the $\mathrm{x}$-ray trigger was varied to observe contrast flow variation at different time intervals postinjection.

We acquired images of contrast flow before and after PED deployment. The PED was partially deployed, resulting in complete coverage of the aneurysmal ostia to accommodate multiple experiments. Images were acquired at $1000 \mathrm{fps}$, yielding 100 images for every 100-ms x-ray exposure period. For comparison with standard angiography, we averaged 40 of these frames to simulate 1 image acquired with an exposure pulse width of $40 \mathrm{~ms}$.

\section{Exposure}

The air kerma at the phantom entrance surface was measured using a 6-mL PTW ionization chamber and electrometer (Model T10023 Unidos; PTW, Freiburg, Germany).

\section{RESULTS AND DISCUSSION}

The air kerma per frame was $12.3 \mu \mathrm{Gy}$ at the phantom entrance surface for each of the 1-ms images acquired at 1000 fps. The air kerma for frames integrated over a sequence of $100 \mathrm{~ms}$ was $1.23 \mathrm{mGy}$ at the entrance surface. This is approximately the dose expected from a DSA run containing 20 frames.

Figure $1(A-C)$ shows 3 images with no PED from a 100-image sequence acquired at $1000 \mathrm{fps}$. Figure $1 D$ shows an image averaged over 40 frames from the same sequence with reduced noise and increased motion blurring. The blur for an actual DSA frame will depend on the pulse width used and the velocity of the blood flow. Noise reduction and blurring over a few tens of a millisecond pulse width would correspond to those of a standard angiography frame. Future studies will be needed to better evaluate any potential effect of noise and noise variation across a nonhomogeneous phantom. Figure 2 shows the corresponding image datasets with a PED partially deployed.

Compared with standard angiographic images, Figs $1 A-C$ and $2 A-C$ demonstrate that additional information, such as detailed flow patterns in the aneurysm region, is visualized in images acquired at $1000 \mathrm{fps}$ due to higher temporal resolution. When no PED flow diverter was deployed, images acquired at 1000 fps (Fig 1A-C and On-line Video 1) clearly show flow into the aneurysm with the development of vortices of contrast in the aneurysmal sac. Images acquired with a PED flow diverter in place (Fig 2A-C and On-line Video 2) show more diffuse flow of contrast into the aneurysm with lower apparent flow velocities and delayed development of vortices in the aneurysm sac. While with the flow phantom reported on here, changes in contrast media streamlines and blob shapes could easily be seen with 1-ms frame intervals, lower imaging speeds might be sufficient under certain circumstances. This will require further study.

For this demonstration, the synchronization between the high-speed detector and the $\mathrm{x}$-ray source was done manually. The 100 -ms exposure time used in this study is a limitation of the $\mathrm{x}$-ray source. 


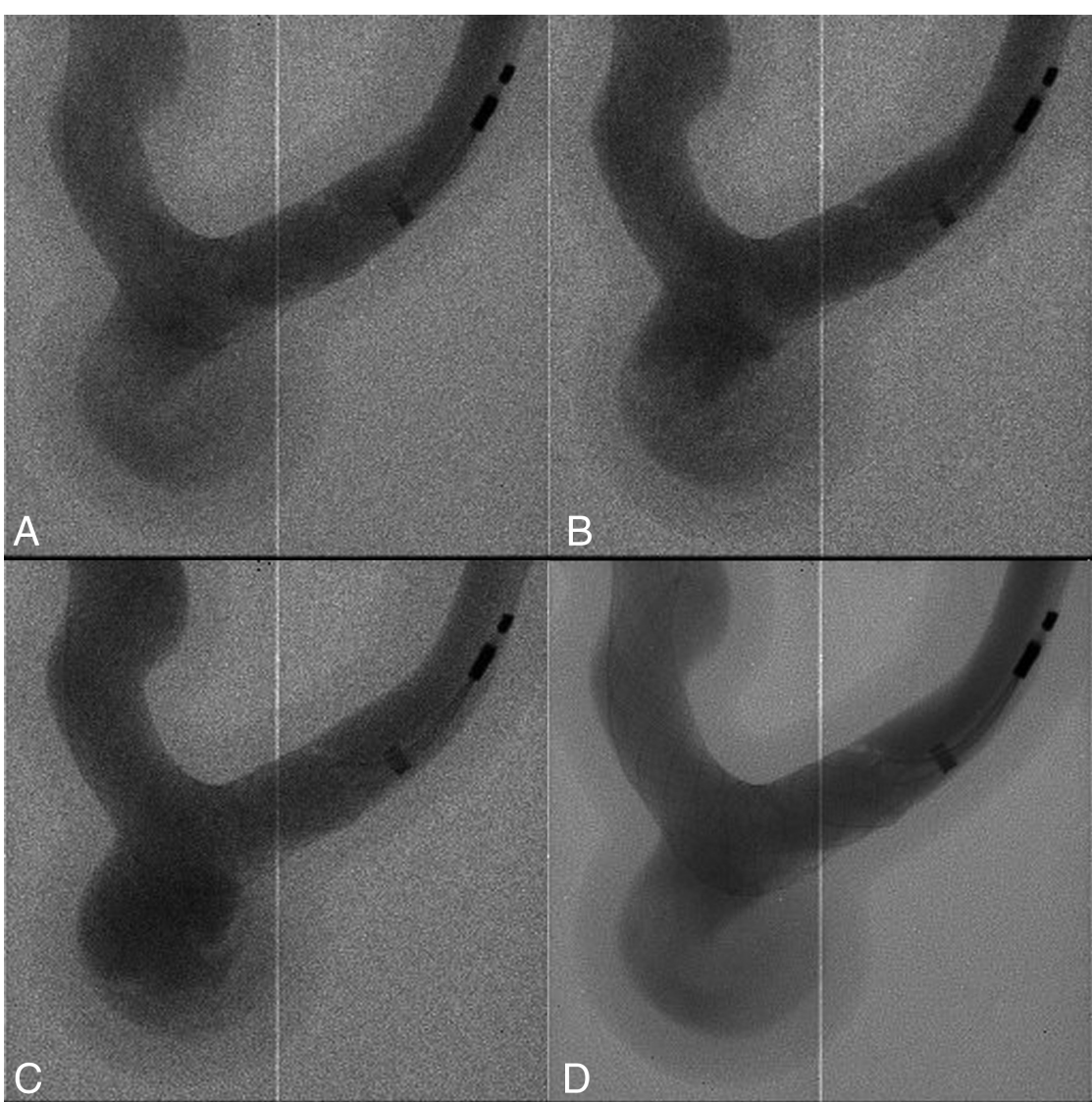

FIG 2. DSA images show flow detail in an internal carotid artery aneurysm model when a PED flow diverter is deployed, acquired at $1000 \mathrm{fps}$ and separated by 25 frames each $(A-C)$, and a single averaged image for one 40-ms $x$-ray pulse $(D)$. For the full sequence, see On-line Video 2. described $^{5}$ and has evolved into the current Hi-Def Detector System (Canon Medical, Tustin, California).

\section{CONCLUSIONS}

Improvements in spatial and temporal resolution of angiography have the potential to significantly improve outcomes by giving clinicians additional real-time insights into the details of flow dynamics, which allow intraprocedural modifications to improve procedural safety and long-term efficacy.

Disclosures: Jordan M. Krebs—RELATED: Grant: Canon Medical Systems Corporation, Comments: research grant for Canon Stroke and Vascular Research Center, where the work took place*; Grants/Grants Pending: Canon Medical Systems Corporation, Comments: research grant for Canon Stroke and Vascular Research Center, where the work took place.* Alok ShankarRELATED: Grant: Canon Medical Systems Corporation, Tokyo, Japan, Comments: research grant. Jason Davies_UNRELATED: Employment: Mr; Grants/ grants pending: CTSR KL2.* Elad I. LevyUNRELATED: Board Membership: Stryker, NeXtGen Biologics, MEDX, Cognition Medical, Endostream Medical, Comments: advisory board; Consultancy: Claret Medical, Gerson Lehrman Group Consulting, Guidepoint Global Consulting, Imperative Care, Medtronic, Rebound Therapeutics, StimMed; Stock/ Stock Options: NeXtGen Biologics, Rapid Medical, Claret Medical, Cognition Medical, Imperative Care, Rebound Therapeutics, StimMed, Three Rivers Medical. Leo Nelson Hopkins-UNRELATED: Board Membership: Claret Medical; Consultancy: Boston Scientific, Abbott, Cordis, Medtronic; Employment:

High-speed imaging, enabled by this new detector, allows clinicians to observe detailed flow patterns in the $3 \mathrm{D}$-printed phantom, including differentiation of flow patterns into the target aneurysm versus the parent vessel. These gains in temporal information could potentially impact treatment decisions by allowing clinicians to assess flow patterns in real-time during a procedure. During treatment, clinicians assess the adequacy of stent placement by looking for indirect signs of flow diversion, such as evaluating wall apposition and looking for contrast stasis within the aneurysm sac. Detailed visualization of changes in flow dynamics could allow clinicians to directly assess factors that have been shown to improve the efficacy of flow diverters, such as decreases in flow vortices. ${ }^{4}$ Other potential clinical uses include the detection of endoleaks near an aneurysm, identification of residual flow that could cause an aneurysm to regrow, blood impingement pattern that could lead to coil compaction, and diversion of jets into an aneurysm at locations where flow may be less intuitive (such as at a bifurcation).

The imager described here is a small-FOV, ROI detector. It is contemplated that within a system that also provides a conventional full FOV capability, a high-speed unit somewhat larger than that shown here could be deployed during an interventional procedure in a manner similar to that of the Micro-Angiographic Fluoroscope (Canon Stroke and Vascular Research Center, University at Buffalo, Buffalo, New York), which has been previously
University at Buffalo Neurosurgery, Jacobs Institute, Ka-leida Health; Payment for Lectures Including Service on Speakers Bureaus: Memorial Healthcare System, Complete Conference Management; Stock/Stock Options: Boston Scientific, Claret Medical, Augmenix, Endomation, Silk Road Medical, Ostial, Apama Medical, StimSox, Photolitec, ValenTx, Axtria, Ocular Therapeutix, The Stroke Project, Comments: financial interests, no money received. Maxim Mokin—UNRELATED: Consultancy: Cerebrotech Medical Systems, Canon Medical Systems Corporation, Imperative Care; Royalties: Oxford University Press. Daniel R. Bednarek—RELATED: Grant: Canon Medical Systems Corporation*; UNRELATED: Grants/Grants Pending: Canon Medical Systems Corporation*; Royalties: Canon Medical Systems Corporation, Comments: Dose Tracking System (DTS) licensing agreement.* Adnan H. Siddiqui-UNRELATED: Consultancy: Amnis Therapeutics, Boston Scientific, Canon Medical Systems USA, Cerebrotech Medical Systems, Cerenovus, Corindus Vascular Robotics, Endostream Medical, Guidepoint Global Consulting, Imperative Care, Integra, Medtronic, MicroVention, Northwest University: Data and Safety Monitoring Board Chair for the HEAT Trial, Penumbra, Q'Apel Medical, Rapid Medical, Rebound Therapeutics, Serenity Medical, Silk Road Medical, StimMed, Stryker, Three Rivers Medical, Vassol, W.L. Gore \& Associates; Stock/Stock Options: Amis Therapeutics, BlinkTBI, Buffalo Technology Partners, Cardinal Consulting, Cerebrotech Medical Systems, Cognition Medical, Endostream Medical, Imperative Q'Apel Medical, Rebound Therapeutics, Rist Neurovascular, Serenity Medical, Silk Road Medical, StimMed, Synchron, Three Rivers Medical, Viseon Spine. Stephen Rudin-RELATED: Grant: Canon Medical Systems Corporation, Comments: An equipment grant from Canon is provided as well as partial research support*; Provision of Writing Assistance, Medicines, Equipment, or Administrative Support: Canon Medical Systems Corporation, Comments: Some x-ray equipment has been provided*; Other: National Institute of Biomedical Imaging and Bioengineering, National Institutes of Health, Comments: Partial support was provided in the past*; UNRELATED: Patents (Planned, Pending or Issued): Canon Medical Systems Corporation, Comments: There is an unrelated patent application pending*; Royalties: Canon Medical Systems Corporation, Comments: totally different project involving dose-tracking system software for clinical angiography equipment. *Money paid to the institution. Care, International Medical Distribution Partners, Neurovascular Diagnostics, 


\section{REFERENCES}

1. Jiang J, Strother C. Computational fluid dynamics simulations of intracranial aneurysms at varying heart rates: a "patient-specific" study. J Biomech Eng 2009;131:091001 CrossRef Medline

2. Shankar A, Krebs J, Bednarek DR, et al. Evaluation of a new photoncounting imaging detector $(\mathrm{PCD})$ with various acquisition modes. Proc SPIE Int Soc Opt Eng 2018;10573 CrossRef Medline

3. Russ M, O'Hara R, Setlur Nagesh SV, et al. Treatment planning for image-guided neuro-vascular interventions using patient-specific 3D printed phantoms. Proc SPIE Int Soc Opt Eng 2015;9417 CrossRef Medline

4. Dholakia R, Sadasivan C, Fiorella DJ, et al. Hemodynamics of flow diverters. J Biomech Eng 2017;139 CrossRef Medline

5. Binning MJ, Orion D, Yashar $\mathrm{P}$, et al. Use of the microangiographic fluoroscope for coiling of intracranial aneurysms. Neurosurgery 2011;69:1131-38 CrossRef Medline 